\title{
Deadtime Correction and Hydride Evaluation for Atom-Probe Data, with Applications for Studies of Nanoscale Grains and Carbon
}

\author{
J. B. Lewis ${ }^{1}$, D. Isheim ${ }^{2}$, C. Floss ${ }^{1}$, T. L. Daulton ${ }^{3}$ and D. N. Seidman ${ }^{2}$ \\ 1. Laboratory for Space Sciences, Physics Department, Washington University, St. Louis, MO, USA. \\ 2. Center for Atom-Probe Tomography, Dept. of Materials Science and Engineering, Northwestern \\ University, Evanston, IL, USA. \\ 3. Center for Materials Innovation, Physics Department, Washington University, St. Louis, MO, USA.
}

Studies of nanoscale volumes by atom-probe tomography (APT) typically involve the measurement of small quantities of trace elements or isotopes present in the region of interest. For example, ongoing studies of the astrophysical origins of meteoritic nanodiamonds (NDs) [1] are based on measuring the ratio of ${ }^{12} \mathrm{C} /{ }^{13} \mathrm{C}$ isotopic abundances. The precision of the APT measurements is ultimately limited by counting statistics. With an APT detection efficiency of $57 \%$ only 13 atoms of ${ }^{13} \mathrm{C}$ would be detected in a ND $(\sim 3 \mathrm{~nm}$ mean diameter [2]) if it were of solar isotopic composition $\left({ }^{12} \mathrm{C} /{ }^{13} \mathrm{C} \sim 89\right)$. Thus, it is essential to minimize all sources of signal loss in order to make statistically significant measurements of the ${ }^{12} \mathrm{C} /{ }^{13} \mathrm{C}$ isotopic ratio. As discussed below, we use correlated ion-detection event analysis, known as multiple-hit analysis, to calculate the APT detector deadtime effect on pairs of $\mathrm{C}$ ions and evaluate hydride formation [3].

Measurements for this study are made with a Cameca LEAP 4000X Si atom-probe tomograph $[1,4]$. In APT ([5] and references therein) we employ a high electric-field bias accompanied by nanosecond laser pulsing to field-evaporate atoms from a 20-100 nm radius needle-shaped microtip and project them onto a microchannel plate detector. By combining point projection field-ion microscopy and time-of-flight mass spectrometry, APT permits reconstruction of the 3-D locations and mass-to-charge-state ratios of atoms and molecules originating from the microtip surface. Sample microtips are composed of NDs sandwiched between sputter-deposited Pt [4] (Fig. 1a-c). The samples are each estimated to contain between 1-100 meteoritic NDs or, for standards, detonation nanodiamonds (DND). We have tailored our multilayer liftout and microtip mounting sample preparation technique, as well as APT experimental parameters, to improve microtip lifetime and stability in the atom probe [e.g., 1, 4].

Detector deadtime effects arise when two or more ions arrive close enough together in space and time to produce only one count in the electronics. We employ multiple-hit analysis [3] to estimate the probability of a pair of ions being lost due to the detector's deadtime. In our work to date $[1,6]$, the ratios measured from standard $\mathrm{ND}$ tips are all lower than the terrestrial value of $\sim 89$, implying a consistent instrumental bias (Fig. 1d). Corrections are being made to the ${ }^{12} \mathrm{C}$ and ${ }^{13} \mathrm{C}$ counts in these data sets to account for deadtime effects. To date, these corrections have reduced but not eliminated this bias.

Hydride formation is another concern. While the mass resolving power in APT is sufficient to distinguish ${ }^{12} \mathrm{C}$ and ${ }^{13} \mathrm{C}$ in all common charge states, it will not distinguish ${ }^{13} \mathrm{C}^{+}$from $\left({ }^{12} \mathrm{C}^{1} \mathrm{H}\right){ }^{+}$. The number of $\left({ }^{1} \mathrm{H}^{+},{ }^{12} \mathrm{C}^{+}\right)$multiple-hit pairs detected are on the same order of magnitude as the total events at $13 \mathrm{Da}$, indicating the potential for hydride interference in the data, but further quantitative analysis of microtips and subvolumes is warranted. Another bias is implied by the fact that the Allende ${ }^{12} \mathrm{C}^{+} /{ }^{13} \mathrm{C}^{+}$ ratios are generally larger than the corresponding Allende ${ }^{12} \mathrm{C}^{++} /{ }^{13} \mathrm{C}^{++}$ratios. This is inconsistent with hydride interference on ${ }^{13} \mathrm{C}^{+}$, which would decrease the ${ }^{12} \mathrm{C}^{+} /{ }^{13} \mathrm{C}^{+}$ratios. We note that the NDs are mixed with an amorphous $\mathrm{C}$ phase [7]. Therefore some of the "nanodiamonds" detected in the Allende data may actually be a different phase of $\mathrm{C}$ that co-evaporates more or less readily with $\mathrm{H}$ than the diamond phase. The proportion of non-diamond $\mathrm{C}$ phases in the analysis volume could vary between the 
Allende and terrestrial detonation samples, so hydride interference, if it occurs, may not have the same effect on all of the Allende data as it does on the standards.

Finally, Allende ratios that are significantly larger or smaller than the DND ratios could be due to inherent isotopic anomalies in the meteoritic NDs, indicative of various presolar origins. While our new standard data set is not yet large enough to be statistically significant, the new Allende ratios generally appear to be larger than the standard ratios.

\section{References:}

[1] PR Heck et al, Meteoritics and Planetary Science (2014), in press.

[2] TL Daulton et al, Geochimica et Cosmochimica Acta 60 (2006), pp. 4853-4872.

[3] DW Saxey, Ultramicroscopy 111 (2011), pp. 473-479.

[4] D Isheim et al, Microscopy and Microanalysis 19 (Suppl 2) (2013), CD974-CD975.

[5] B Gault et al, "Atom Probe Microscopy”, ed. R Hull et al. (Springer, New York).

[6] JB Lewis et al, 45 ${ }^{\text {th }}$ Lunar and Planetary Science Conference (2014) \#2607.

[7] RM Stroud et al, Astrophysical Journal Letters 738 (2011), L27-L31.

[8] This work is supported by NASA grant NNX13AF53G (C.F.). We are indebted to Dr. Frank

Stadermann whose ideas and enthusiasm this research is based upon. We thank R.S. Lewis for providing the NDs from the Allende meteorite. The LEAP tomograph at NUCAPT was purchased and upgraded with funding from NSF-MRI and ONR-DURIP programs. Instrumentation at NUCAPT was supported by the Initiative for Sustainability and Energy at Northwestern. This research made use of the EPIC facility and Shared Facilities at the Materials Research Center, supported by the MRSEC program (DMR-0520513 and 1121262) of NSF.
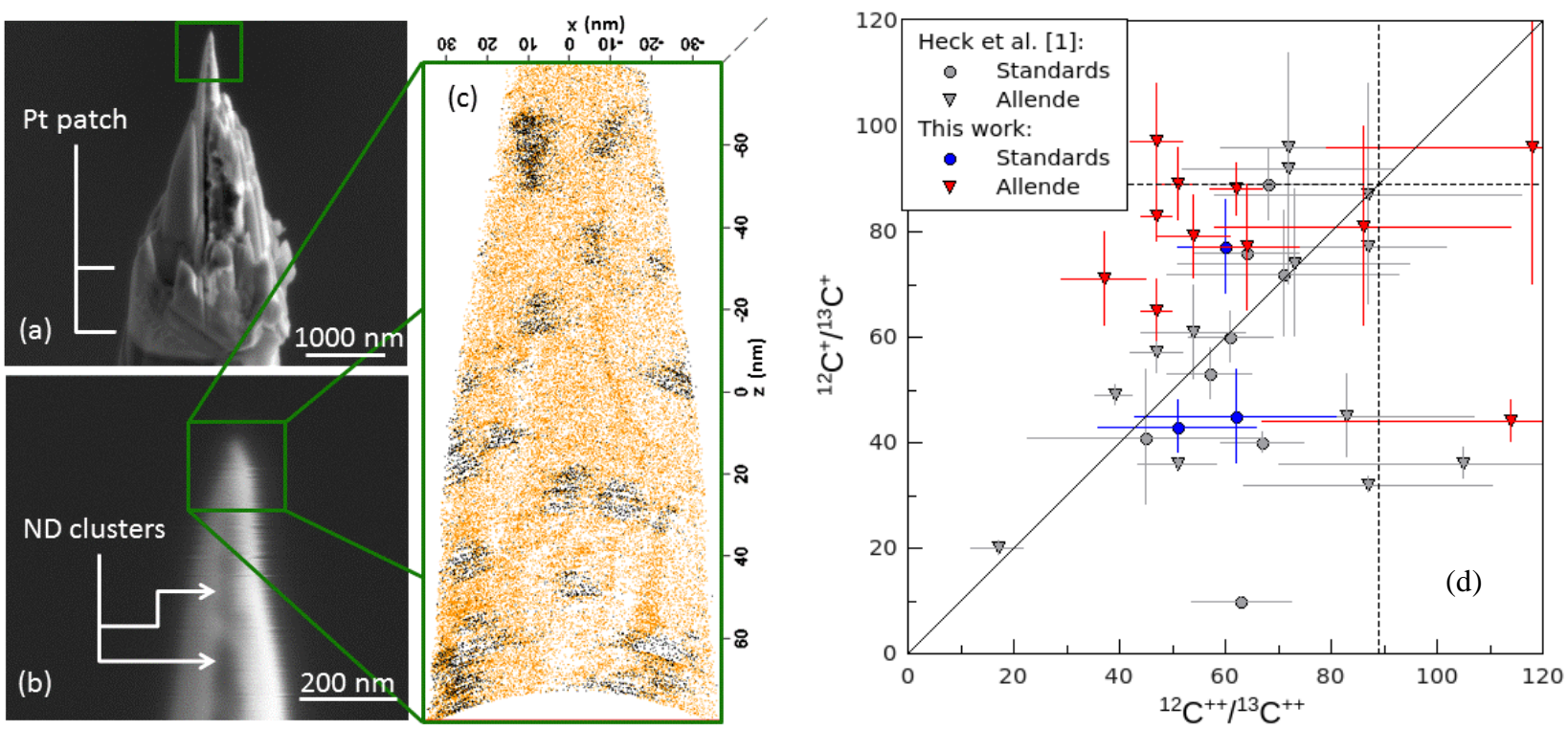

Figure 1. (a) A microtip attached to a $\mathrm{Si}$ micropost by a Pt patch. (b) The same tip at higher magnification with dark ND clusters in the deposition layer. (c) Cross-section of APT reconstruction exhibiting individual NDs (black dots are $\mathrm{C}$ atoms) in a Pt matrix (orange dots). (d) Graphical summary of ${ }^{12} \mathrm{C} /{ }^{13} \mathrm{C}$ isotopic ratios determined by APT separately for singly- and doubly-charged $\mathrm{C}$ ions. Each data point represents the ratios of counts from an individual microtip. Errors are 1o, based on counting statistics. Dashed lines mark the terrestrial ${ }^{12} \mathrm{C} /{ }^{13} \mathrm{C}$ ratio of $\sim 89$. The solid diagonal line indicates where equal ratios for singly- and doubly-charged $\mathrm{C}$ ions lie. 